\begin{tabular}{|l|l|}
\hline Research Article & Open Access \\
\hline
\end{tabular}

\title{
Differential Antioxidant Mechanisms of Rice Plants in Response to Oxyfluorfen and Paraquat
}

\author{
Jin-Gil Kim and Sunyo Jung* \\ School of Life Sciences and Biotechnology, Kyungpook National University, Daegu 702-701, Korea \\ (Received on September 9, 2013; Revised onSeptember 12, 2013; September 16, 2013)
}

\begin{abstract}
The mechanisms of resistance to oxyfluorfen $(\mathrm{OF})$ and paraquat $(\mathrm{PQ})$ were investigated in rice plants. Examination of the concentration dependence of oxyfluorfen- or paraquat-induced increase in conductivity showed that conductivities in the OF- and PQ-treated leaf squares were increased with $0.1 \mu \mathrm{M}$ oxyfluorfen and $0.01 \mu \mathrm{M}$ paraquat and further increased with higher concentrations. The levels of conductivity were approximately 10-times higher in the PQ-treated plants than in the OF-treated plants, indicating that the PQ-treated plants suffered more severe photodynamic damage than the OF-treated plants. The photooxidative stress caused by foliar application of either $50 \mu \mathrm{M}$ oxyfluorfen or $100 \mu \mathrm{M}$ paraquat increased the enzyme activities of ascorbate peroxidase and peroxidase 1 day after the herbicide treatments and then further increased their enzyme activities 2 days after the treatments. The activities of catalase began to increase 2 days after the oxyfluorfen and paraquat treatments. These antioxidant enzymes appear to play an essential part of defense mechanisms against oxyfluorfen and paraquat. Our results demonstrate that paraquat caused more severe oxidative stress, as indicated by a greater change in conductivity, thereby resulting in greater increases in antioxidant responses in plants, compared with those of oxyfluorfen.
\end{abstract}

Key words: Antioxidant enzyme, Oxidative stress, Oxyfluorfen, Paraquat, Rice plants

\section{Introduction}

Reactive oxygen species (ROS) including superoxide radical $\left(\cdot \mathrm{O}_{2}^{-}\right)$and singlet oxygen $\left({ }^{1} \mathrm{O}_{2}\right)$ can result from cellular exposure to various environmental stresses such as UV light and other forms of radiation, pathogens, ozone and temperature fluctuations (Scandalios, 1993; Suzuki et al., 2012). Oxyfluorfen, a diphenylether herbicide, is inhibitor of protoporphyrinogen oxidase (PPO) which catalyzes the step leading to the formation of protoporphyrin IX (Proto IX) from protoporphyrinogen IX (Protogen IX) (Jung and Back, 2005; Nandihalli et al., 1992). This inhibition results in the accumulation of Protogen IX which diffuses to the cytoplasm and is oxidized to Proto IX via peroxidase-like enzymes in membrane. Cytoplasmic Proto IX is a potent photosensitizer resulting in the formation of ROS, causing peroxidation of membrane lipids and cell death (Jacobs et al., 1991; Lee et al., 1993). Herbicides that block electron transport or act as alternative electron acceptors and increase the flow of electrons to oxygen also significantly increase the production of ROS (Powles and Cornic, 1987). The bipyridylium herbicide paraquat is a nonselective contact

\footnotetext{
*Corresponding author:

Phone) +82-53-950-7364, Fax) +82-53-943-6925

E-mail)sjung@knu.ac.kr
}

herbicide that acts by intercepting electrons from the photosynthetic electron transport chain at photosystem I. This reaction results in the production of bipyridyl radicals that readily react with $\mathrm{O}_{2}$ to produce $\cdot \mathrm{O}_{2}^{-}$and then, through a series of reactions, produce $\mathrm{H}_{2} \mathrm{O}_{2}$ and the hydroxyl radical. These toxic oxygen species cause extensive lipid peroxidation leading to loss of cell membrane integrity and rapid desiccation (Babbs et al., 1989; Kunert and Dodge, 1989). The generation of ROS contributes to the toxic effects of these herbicides. This perturbation can cause excess ROS and necessitate additional defenses.

The ROS themselves are proposed to trigger necrosis, programmed cell death as well as the induction of protective mechanisms (Scandalios, 1993; Suzuki et al., 2012). The metabolism of ROS is dependent on functionally related antioxidant enzymes, particularly hemoproteins including catalase (CAT), ascorbate peroxidase (APX) and peroxidase (POD), which destroy $\mathrm{H}_{2} \mathrm{O}_{2}$ (Foyer and Shigeoka, 2011; Scandalios, 1993). Enzymatic antioxidant systems provide protection against the toxic effects of ROS. APX, together with SOD constitutes the major defense system against ROS in chloroplasts (Dalton et al., 1986; Foyer and Shigeoka, 2011).

The differential herbicidal effects of diphenylether herbicide and paraquat were previously studied in tobacco leaves of varying ages (Kuk et al., 2003); however, the differential mechanism for these herbicides was not clearly 
elucidated in major crop plants and particularly in antioxidant responses to oxidative stresses caused by different herbicidal actions. In our study, rice plants treated either with oxyfluorfen or paraquat resulted in a substantial increase in oxidative stress, as indicated by dehydration and necrotic spots on leaves. The similarities raised the question of whether oxyfluorfen exhibits the same influence not only on oxidative stress but also on antioxidant responses as in paraquat-treated plants. A concurring alteration at enzymatic antioxidant levels against excess light and possible photooxidative stress was compared between oxyfluorfen (OF)-treated plants and paraquat (PQ)-treated plants. We established the differential antioxidant mechanisms in response to oxidative stress caused by two different herbicidal actions, photodynamic damage and photosynthetic inhibition, in rice plants treated with oxyfluorfen and paraquat, respectively.

\section{Materials and Methods}

\section{Plant material and herbicide application}

Rice seeds (Oryza sativa cv. Dongjin) were immersed in tap water for 2 days to induce germination, and then potted in a tray filled with greenhouse compost (Boonong Soil, Seoul, Korea). Seedlings were potted in paddy soil and grown in a greenhouse at $30^{\circ} \mathrm{C}$. The plants were used 4 weeks after sowing. For the conductivity test, we used technical-grade oxyfluorfen (98.6\%) (Kyungnong, Kyungju, Korea). Commercially available oxyfluorfen $\left(\mathrm{Goal}^{\circledR}\right)$ and paraquat (Sigma) were used for a foliar application test. Plants were sprayed with herbicide using either oxyfluorfen or paraquat. For foliar application, plants were placed in the dark immediately after treatment for $12 \mathrm{~h}$ and then exposed to light. Leaf samples were taken $4 \mathrm{~h}$ and $28 \mathrm{~h}$ after light exposure for day 1 and day 2 leaf samples, respectively.

\section{Electrolyte leakage assay}

The rice leaf tissues were treated with oxyfluorfen and paraquat as described previously (Kenyon et al., 1985; Lee et al., 1995) by cutting 4-mm leaf squares ( $0.1 \mathrm{~g} \mathrm{FW})$ with a razor blade and then placing them in a $6-\mathrm{cm}$ diameter polystyrene Petri dish containing $5 \mathrm{ml}$ of $1 \%$ sucrose and $1 \mathrm{mM}$ MES ( $\mathrm{pH}$ 6.5) with or without the herbicide dissolved in acetone. The controls contained the same amount of the solvent without the herbicide. The final concentration of acetone in all dishes was $1 \%(\mathrm{v} / \mathrm{v})$. The tissues were incubated with various concentrations of oxyfluorfen or paraquat in a growth chamber at $25^{\circ} \mathrm{C}$ in darkness for $12 \mathrm{~h}$, and then exposed to continuous white light at $250 \mu \mathrm{mol} \mathrm{m} \mathrm{m}^{-2}$ PPFD (photosynthetic photon flux density) for $12 \mathrm{~h}$. Cellular electrolyte leakage was determined periodically by the detection of electrolyte leakage into the bathing medium using a conductivity meter (Cole-Parmer Instruments) as described in Lee et al. (1995). Because of differences in the background conductivity of different treatment solutions, the results were expressed as changes in conductivity upon exposure to light.

\section{Extraction of soluble protein}

For antioxidant enzyme assays, frozen leaves $(0.25 \mathrm{~g})$ were macerated to fine powder in a mortar under liquid $\mathrm{N}_{2}$. Soluble proteins were extracted by homogenizing the powder in $2 \mathrm{ml}$ of $100 \mathrm{mM}$ potassium phosphate buffer, $\mathrm{pH}$ 7.5 , containing $2 \mathrm{mM}$ EDTA, $1 \%$ polyvinylpolypyrrolidone40 , and $1 \mathrm{mM}$ phenylmethylsulfonyl fluoride. Insoluble material was removed by centrifugation at $15,000 \times g$ for 20 $\min$ at $4^{\circ} \mathrm{C}$. Since maintenance of consistent catalase activity was found to require the presence of DTT, an aliquot of each sample containing $10 \mathrm{mM}$ DTT to be used for catalase spectrometric assay.

\section{Assays for antioxidant enzyme activities}

Ascorbate peroxidase (APX) activity was measured spectrophotometrically by monitoring the decline in $\mathrm{A}_{290}$ as ascorbate was oxidized, using the method of Chen and Asada (1989). The 3-ml reaction volume contained $100 \mathrm{mM}$ potassium phosphate buffer ( $\mathrm{pH} 7.5), 0.5 \mathrm{mM}$ ascorbate, and $0.2 \mathrm{mM} \mathrm{H}_{2} \mathrm{O}_{2}$ at $25^{\circ} \mathrm{C}$. Total Catalase (CAT) activity was determined spectrophotometrically in a $3 \mathrm{ml}$ volume containing $50 \mathrm{mM}$ potassium phosphate buffer, $\mathrm{pH}$ 7.0, containing $20 \mathrm{mM} \mathrm{H}_{2} \mathrm{O}_{2}$ by monitoring $\mathrm{H}_{2} \mathrm{O}_{2}$ destruction at $240 \mathrm{~nm}$ (Beers and Sizer, 1952). Peroxidase (POD) activity was determined specifically with guaiacol at $470 \mathrm{~nm}$ following the method of Egley et al. (1983). The reaction mixture contained $40 \mathrm{mM}$ potassium phosphate buffer $(\mathrm{pH}$ 6.9), $1.5 \mathrm{mM}$ guaiacol, and $6.5 \mathrm{mM} \mathrm{H}_{2} \mathrm{O}_{2}$ in a 3-ml volume.

\section{Results and Discussion}

\section{Effect of oxyfluorfen and paraquat treatment on conductivity of rice plants}

Both oxyfluorfen and paraquat are photooxidative stressinduced herbicides that block PPO enzyme and subvert electron transport, respectively (Babbs et al., 1989; Kunert and Dodge, 1989; Nandihalli et al., 1992; Powles and Cornic, 1987), and thereby resulted in desiccation and necrotic symptoms in rice plants treated with either oxyfluorfen or paraquat. We have used electroleakage as an indicator of the appearance of herbicidal response of oxyfluorfen and paraquat in the treated rice plants. The necrosis of leaf squares upon oxyfluorfen (OF) and paraquat (PQ) treatments was associated with an increase in conductivity, which indicates cellular leakage (Figs. 1 and 2 ). Conductivities in the OF-treated plants were increased 


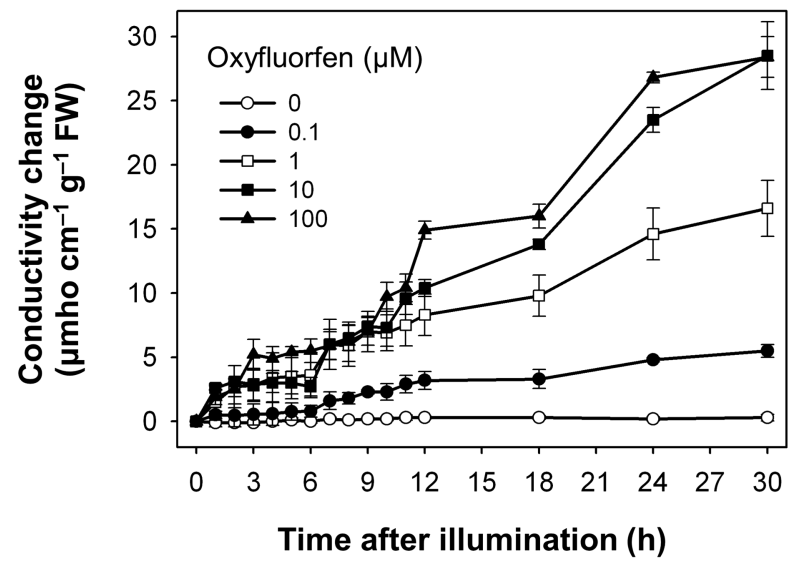

Fig. 1. Effect of oxyfluorfen on cellular electrolyte leakage from rice leaf squares. Conductivity, which reflected electrolyte leakage, was measured in the bathing solution after leaf segments were treated with various concentrations of oxyfluorfen. Tissues were exposed to continuous white light at $250 \mu \mathrm{mol} \mathrm{m} \mathrm{m}^{-2} \mathrm{~s}^{-1} \mathrm{PPFD}$ for 30 hour following a 12 hour dark incubation. The data represent the mean \pm S.E. of three replicates.

with $0.1 \mu \mathrm{M}$ oxyfluorfen and were further increased with higher oxyfluorfen concentrations (Fig. 1). The conductivities were drastically increased until $24 \mathrm{~h}$ after OF treatment and then the rates of conductivity increase were slowed down. Paraquat incubation with light for $30 \mathrm{~h}$ also resulted in necrosis on leaf squares of rice plants. Conductivities in the PQ-treated plants were slightly increased with $0.1 \mu \mathrm{M}$ paraquat and were further increased with higher paraquat concentrations (Fig. 2). The conductivities were increased

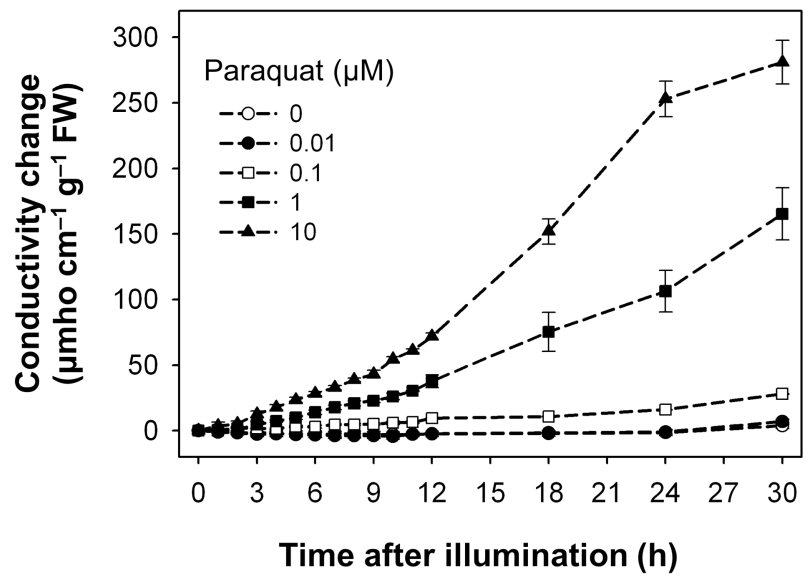

Fig. 2. Effect of paraquat on cellular electrolyte leakage from rice leaf squares. Conductivity, which reflected electrolyte leakage, was measured in the bathing solution after leaf segments were treated with various concentrations of paraquat. Tissues were exposed to continuous white light at $250 \mu \mathrm{mol} \mathrm{m} \mathrm{s}^{-2}$ PPFD for 30 hour following a 12 hour dark incubation. The data represent the mean \pm S.E. of three replicates. continuously during $30 \mathrm{~h}$ of illumination. The levels of conductivity were approximately 10-times higher in the PQtreated plants than in the OF-treated plants, indicating that the PQ-treated plants suffered more severe photodynamic damage than the OF-treated plants. With oxyfluorfen treatment using leaf squares, rice plants showed the increased conductivity levels, which may be due to a drastic accumulation of Proto IX in oxyfluorfen-treated plants. The necrotic phenotype of peroxidizing herbicide-treated plants displays leaf desiccation, veinal necrosis, and leaf deformation (Böger and Wakabayashi, 1999; Jung et al., 2004). The PQ-treated plants appears to increase their conductivity mainly due to the inhibited dissipation of toxic $\mathrm{H}_{2} \mathrm{O}_{2}$ through abnormal photosynthetic electron transport.

\section{Activities of antioxidant enzymes upon foliar application of oxyfluorfen and paraquat}

Foliar application of both $50 \mu \mathrm{M}$ oxyfluorfen and $100 \mu \mathrm{M}$ paraquat showed slight necrotic symptom on leaves by 1 day after those treatments and then severe necrosis and desiccation within 2 days after the treatments. However, the rice plants treated with paraquat suffered faster dehydration symptom but fewer necrotic spots, compared to the plants treated with oxyfluorfen. It has been proposed that the mechanism of herbicide resistance is a result of increased levels of enzymes that detoxify active $\mathrm{O}_{2}$ species (Jung and Back, 2005). Contribution of enzymatic antioxidant responses, which are mediated by increasing amounts of ROS upon herbicidal treatment, was compared in OF- and PQ-treated rice plants. Total activities of all antioxidant enzymes including APX, CAT and POD increased upon both oxyfluorfen and paraquat treatment compared with those of untreated control plants (Figs. 3-5). At 1 day after the administration of $50 \mu \mathrm{M}$ oxyfluorfen, rice plants did not exhibit any noticeable change in enzyme activities of APX (Fig. 3). APX activity was noticeably increased in rice plants at 2 days after oxyfluorfen treatment. Total APX activity exhibited 3-fold increase 1 day after $100 \mu \mathrm{M}$ of paraquat treatment and further increased 2 days after the treatment (Fig. 3). APX utilizes ascorabate as a specific electron donor to reduce $\mathrm{H}_{2} \mathrm{O}_{2}$ to water with the concomitant generation of monodehydroascorbate, a univalent oxidant of ascorbate (Foyer and Shigeoka, 2011). In the OF- and PQtreated plants, total activities of CAT were not noticeably changed at early stages of the treatments, but considerably increased 2 days after the herbicide treatments (Fig. 4). The maximum increases in CAT activity were observed on day 2 in the PQ-treated plants. CAT is known to take part in an efficient protective role against oxidative stress (Chaoui et al., 1997). In response to the two herbicide treatments, total POD activity increased at 1 day after the treatment and then increased further in both OF- and PQ-treated plants, in comparison to untreated control plants (Fig. 5). The paraquat 


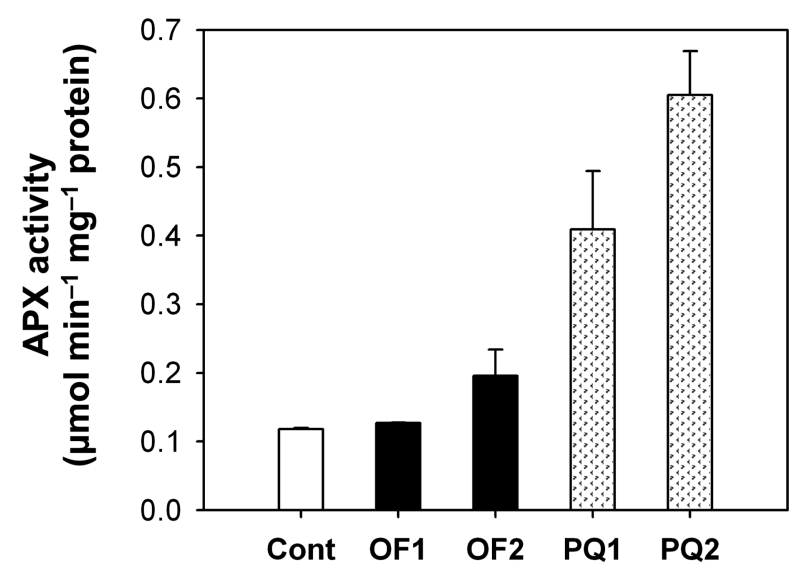

Fig. 3. Effects of oxyfluorfen and paraquat on enzyme activities of ascorbate peroxidase (APX). The 4-week-old rice plants were subjected to the foliar application of either $50 \mu \mathrm{M}$ oxyfluorfen or $100 \mu \mathrm{M}$ paraquat. Cont: untreated control; OF1: 1 day after oxyfluorfen treatment; OF2: 2 days after oxyfluorfen treatment; PQ1: 1 day after paraquat treatment; PQ2: 2 days after paraquat treatment. The data represent the mean $\pm \mathrm{SE}$ of 6 plants from two independent experiments.

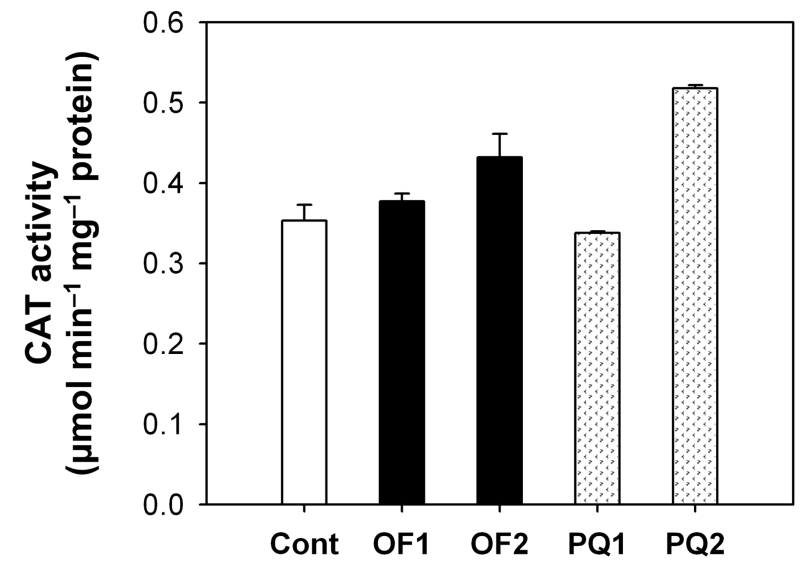

Fig. 4. Effects of oxyfluorfen and paraquat on enzyme activities of catalase (CAT). The 4-week-old rice plants were subjected to the foliar application of either $50 \mu \mathrm{M}$ oxyfluorfen or $100 \mu \mathrm{M}$ paraquat. Cont: untreated control; OF1: 1 day after oxyfluorfen treatment; OF2: 2 days after oxyfluorfen treatment; PQ1: 1 day after paraquat treatment; PQ2: 2 days after paraquat treatment. The data represent the mean $\pm \mathrm{SE}$ of 6 plants from two independent experiments.

treatment resulted a greater increase in POD activity than that of the oxyfluorfen treatment. The enzymatic removal of $\mathrm{H}_{2} \mathrm{O}_{2}$ by POD and CAT seems to be the dominant pathway during oxyfluorfen- and paraquat-induced enzymatic scavenging system in rice. Overall, paraquat treatment resulted in marked increase in antioxidant enzyme activities in comparison to those of oxyfluorfen treatment, implying that the PQ-treated plants developed stronger defense responses against the

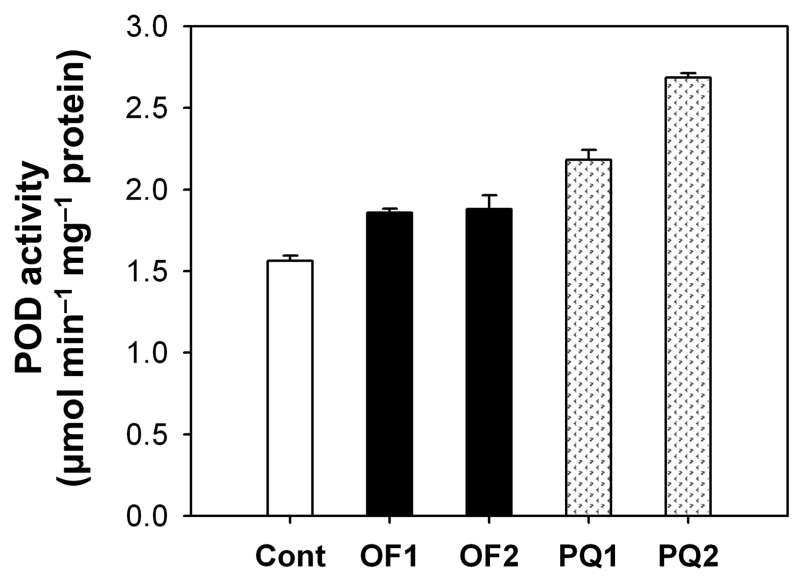

Fig. 5. Effects of oxyfluorfen and paraquat on enzyme activities of peroxidase (POD). The 4-week-old rice plants were subjected to the foliar application of either $50 \mu \mathrm{M}$ oxyfluorfen or $100 \mu \mathrm{M}$ paraquat. Cont: untreated control; OF1: 1 day after oxyfluorfen treatment; OF2: 2 days after oxyfluorfen treatment; PQ1: 1 day after paraquat treatment; $\mathrm{PQ} 2: 2$ days after paraquat treatment. The data represent the mean $\mathrm{SE}$ of 6 plants from two independent experiments.

oxidative stress.

ROS produced in plants are normally detoxified by enzymatic and non-enzymatic antioxidants present in all cellular compartments, especially at sites where oxygen radicals are usually photogenerated (Foyer et al., 1994; Jung, 2011). Increased levels of the enzymes that detoxify active $\mathrm{O}_{2}$ species have been implicated in protective mechanism. The photooxidative stress caused by either oxyfluorfen or paraquat appears to trigger the dissipative process, enzymatic antioxidants including APX, CAT, and POD. Various antioxidant enzymes appear to play an essential part of defense mechanisms against oxyfluorfenand paraquat-induced oxidative stress. The combined action of an $\mathrm{H}_{2} \mathrm{O}_{2}$-scavenging enzyme, CAT and POD, is critical in mitigating the effects of oxidative stress (Foyer and Shigeoka, 2011; Scandalios, 1993; Suzuki et al., 2012). On the basis of differences in antioxidant responses of rice plants to the two different herbicide applications, we suggest that the mechanism of resistance to oxyfluorfen in OFtreated plants must differ from that in PQ-treated plants.

\section{Differential antioxidant mechanisms between oxyfluorefn and paraquat}

The way to achieve the herbicidal symptoms appears to be different between the two herbicides, oxyfluorfen and paraquat. Oxyfluorfen mainly produces ${ }^{1} \mathrm{O}_{2}$, whereas paraquat produces $\mathrm{H}_{2} \mathrm{O}_{2}$ in plants (Babbs et al., 1989; Jacobs et al., 1991; Kunert and Dodge, 1989; Lee et al., 1993). The mechanism for plant resistance to oxidative stress may 
involve increased antioxidant activities, thereby resulting in alleviated damage in plants; however, this was not clearly elucidated. When plants are exposed to high-intensity light, high or low temperatures, or ozone as well as herbicides, more ROS are generated than the scavenging mechanisms can detoxify (Alscher et al., 1997). In our study, an improved adaptive capacity of the antioxidative pathway for the detoxification of oxidative stress was generated during the oxyfluorfen and paraquat actions. Resistance to oxyfluorfen and paraquat in the rice plants is a result of the increased activities of APX, CAT, and POD in the herbicide-treated plants. However, this was not sufficient to overcome the oxidative stress, thereby resulting in severe herbicidal response in both OF- and PQ-treated plants, particularly in the PQ-treated plants. The similarity of the results obtained with paraquat and oxyfluorfen suggests that the antioxidant enzymes might provide resistance to the oxidative stress caused by both herbicides in rice plants. However, the results of these experiments demonstrate that paraquat caused not only more severe oxidative stress, as indicated by a greater change in conductivity, but also greater increases in antioxidant responses, compared with those of oxyfluorfen. This may indicate that the faster dehydration in PQ-treated plants led to greater alteration in plant defense system than the severe necrotic spots in OF-treated plants. We investigated differential oxidative stress and antioxidant mechanisms induced by oxyfluorfen and paraquat; how plants respond to handle oxyfluorfen and paraquat in which antioxidant enzymes are involved in these responses, and how these processes help protect crops against herbicides and consider the importance of these processes to successful weed management.

\section{Acknowledgement}

This research was supported by Kyungpook National University Research Fund, 2012.

\section{References}

Alscher, R.G., Donahue, J.L. and Cramer, C.L. 1997. Reactive oxygen species and antioxidants: relationships in green cells. Physiol. Plant. 100:224-233.

Babbs, C.F., Pham, J.A. and Coolbaugh, R.C. 1989. Lethal hydroxyl radical production in paraquat-treated plants. Plant Physiol. 90:1267-1270.

Beers, P.F. and Sizer, I.W. 1952. A spectrophotometric assay measuring the breakdown of hydrogen peroxide by catalase. J. Biol. Chem. 195:133-138.

Böger, P. and Wakabayashi, K. 1999. Peroxidizing Herbicides, Springer, Berlin, Heidelberg, Germany.

Chaoui, A., Mazhoudi, S., Ghorbal, M.H. and Ferjani, E.E. 1997.
Cadmium and zinc induction of lipid peroxidation and effects on antioxidant enzyme activities in bean (Phaseolus vulgaris L.). Plant Sci. 127:139-147.

Chen, G.X. and Asada, K. 1989. Ascorbate peroxidase in tea leaves: occurrence of two isozymes and the differences in their enzymatic and molecular properties. Plant Cell Physiol. 30:987-998.

Dalton, D., Russel, S., Hanus, F., Pascoe, G. and Evans, H. 1986. Enzymatic reactions of ascorbate and glutathione that prevent peroxide damage in soy bean root nodules. Proc. Natl. Acad. Sci. USA 83:3811-3815.

Egley, G.H., Paul Jr., R.N., Vaughn, K.C. and Duke, S.O. 1983. Role of peroxidase in the development of water-impermeable seed coats in Sida spinosa L. Planta 157:224-232.

Foyer, C.H., Lelandais, M. and Kunert, K.J. 1994. Photooxidative stress in plants. Physiol. Plant. 92:696-717.

Foyer, H.C. and Shigeoka, S. 2011. Understanding oxidative stress and antioxidant functions to enhance photosynthesis. Plant Physiol. 155:93-100.

Jacobs, J.M., Jacobs, N.J., Sherman, T.D. and Duke, S.O. 1991. Effects of diphenyl ether herbicides on oxidation of protoporphyrinogen to protoporphyrin in organellar and plasma membrane enriched fractions of barley. Plant Physiol. 97:197-203.

Jung, S. 2011. Photodynamic stress-induced nonenzymatic antioxidant responses in transgenic rice overexpressing 5-aminolevulinic acid synthase. Kor. J. Weed Sci. 31:323-329. (In Korean)

Jung, S. and Back, K. 2005. Herbicidal and antioxidant responses of transgenic rice overexpressing Myxococcus xanthus protoporphyrinogen oxidase. Plant Physiol. Biochem. 43:423-430.

Jung, S., Lee, Y., Yang, K., Lee, S.B., Jang, S.M., Ha, S.B. and Back, K. 2004. Dual targeting of Myxococcus xanthus protoporphyrinogen oxidase into chloroplasts and mitochondria and high level oxyfluorfen resistance. Plant Cell Environ. 27:14361446.

Kenyon, W.H., Duke, S.O. and Vaughn, K.C. 1985. Sequence of effects of acifluorfen on physiological and ultrastructural parameters in cucmber cotyledon discs. Pestic. Biochem. Physiol. 24:240-250.

Kuk, Y.I., Shin, J.S., Chung J.S., Kwon, O.D., Kim, D.G., Han, O.S. and Guh, J.O. 2003. Differential tolerances and their mechanisms on the response of tobacco (Nicotiana tabacum L.) leaves of varing ages to protoporphyrinogen oxidase-inhibiting herbicides and paraquat. Kor. J. Weed Sci. 23:100-111. (In Korean)

Kunert, K.J. and Dodge, A.D. 1989. Herbicide-induced radical damage and antioxidative systems, pp. 45-63. In: Böger, P. and Sandmann, G. (Eds.). Target Sites of Herbicide Action. CRC Press Inc., Boca Raton, FL., USA.

Lee, H.J., Duke, M.V., Birk, J.H., Yamamoto, M. and Duke, S.O. 1995. Biochemical and physiological effects of benzheterocycles and related compounds. J. Agric. Food Chem. 43:27222727. 
Lee, H.J., Duke, M.V. and Duke, S.O. 1993. Cellular locxalization of protoporphyrinogen-oxidizing activities of etiolated barley (Hordeum vulgare L.) leaves. Plant Physiol. 102:881-889.

Nandihalli, U.B., Duke, M.V. and Duke, S.O. 1992. Relationships between molecular properties and biological activities of $O$ phenyl pyrrolidino- and piperidinocarbamate herbicides. J. Agric. Food Chem. 40:1993-2000.

Powles, S.B. and Cornic, G. 1987. Mechanism of paraquat resis- tance in Hordeum glaucum. I. Studies with isolated organelles and enzymes. Aust. J. Plant Physiol. 14:81-89.

Scandalios, J.G. 1993. Oxygen stress and superoxide dismutases. Plant Physiol. 101:7-12.

Suzuki, N., Koussevitzky, S., Mittler, R. and Miller, G. 2012. ROS and redox signalling in the response of plants to abiotic stress. Plant Cell Environ. 35:259-270. 